\title{
Electrocardiographic and electrophysiological characteristics of idiopathic ventricular arrhythmias with acute successful ablation at the anterior portion of the mitral annulus
}

\author{
Chengye Di \\ TEDA International Cardiovascular Hospital https://orcid.org/0000-0002-7777-574X \\ Konstantinos Letsas \\ Peng Gao \\ TEDA International Cardiovascular Hospital \\ Qun Wang \\ TEDA International Cardiovascular Hospital \\ Yanxi Wu \\ TEDA International Cardiovascular Hospital \\ Wenhua Lin ( $\square$ linwenhuatich@163.com) \\ TEDA International Cardiovascular Hospital https://orcid.org/0000-0002-0145-1677
}

Evangelismos Athens General Hospital Cardiology: Geniko Nosokomeio Athenon O Euangelismos Kardiologiko

Research article

Keywords: Catheter ablation, electrophysiology mapping, electrograms, ventricular arrhythmia, mitral annular, anterior portion

Posted Date: January 29th, 2021

DOl: https://doi.org/10.21203/rs.3.rs-156734/v1

License: (1) This work is licensed under a Creative Commons Attribution 4.0 International License. Read Full License

Version of Record: A version of this preprint was published at BMC Cardiovascular Disorders on August 18th, 2021. See the published version at https://doi.org/10.1186/s12872-021-02205-0. 


\section{Abstract}

Background: We sought to clarify the electrocardiographic and electrophysiological characteristics of ventricular arrhythmias (VAs), including idiopathic ventricular tachycardia (VT) and premature ventricular contractions (PVCs), with acute successful radiofrequency catheter ablation (RFCA) at the anterior portion of the mitral annulus (AP-MA).

Methods and Results: Among 437 consecutive patients who presented with VAs for RFCA, twenty-six patients with acute successful RFCA at the AP-MA were included in this study. The ratio of the amplitude of the first positive peak (if present) vs. the nadir in the unipolar electrogram (EGM) was $0.00-0.03(0.00)$ at the acute successful RFCA site. The time interval between the QRS onset to the maximum descending slope (D-Max) in the unipolar EGM (QRS - Uni) were $18.8 \pm 13.6 \mathrm{~ms}$. With bipolar mapping, the V-QRS interval was 3.75 - $17.3(11) \mathrm{ms}, 6(23.1 \%)$ patients showed earliest V-QRS interval of 0 ms, and the other 20 patients $(76.9 \%)$ showed V-QRS interval of 10-54 ms. The RFCA start-to-effect time was $14.1 \pm 7.2$ seconds in 23 patients (88.5\%). In the remaining 3 patients (11.5\%), the mean duration of successful RFCA were not well determined due to infrequent nature of clinical VAs during RFCA. Early (within 3 days) and late (one-year) recurrence rates were $23.1 \%$ (6 patients) and $26.9 \%$ (7 patients), respectively. The VAs disappeared 3 days later due to delayed RFCA efficacy in 2 patients (7.7\%). No complications occurred during RFCA or the one-year follow up.

Conclusions: AP-MA VAs are a rare but distinct subgroup of VAs. Bipolar and unipolar EGM features can help to detect the optimal RFCA site, and the QRS - Uni interval may work as a marker for guiding RFCA.

\section{Background}

Most idiopathic ventricular arrhythmias (VAs), including idiopathic ventricular tachycardia (VT) and premature ventricular contractions (PVCs), have a right ventricular outflow tract (RVOT) or left ventricular outflow tract (LVOT) origin, but some may arise from various anatomical sites, including the right or left ventricular epicardial site, the aortic sinus cusp (ASC), the aortomitral continuity (AMC), around the anterior portion of the mitral annulus (AP-MA), and other sites [1]. Radiofrequency catheter ablation (RFCA) has emerged as a treatment for VAs, with a fairly high success rate. Recently, several cases of VAs have been reported to be successfully ablated at the MA and the AP-MA in close proximity to the AMC [2,3]. The QRS morphology on electrocardiogram (ECG) of the VAs originating from the ASC, AMC, left ventricular summit area or AP-MA can mimic each other because of their anatomical vicinity [4]. However, little is known about the prevalence, ECG and electrophysiological (EP) characteristics, efficacy of RFCA and follow-up findings of AP-MA VAs. This study was undertaken to clarify these points.

\section{Methods}

\section{Study participants}

Among 437 consecutive patients who presented with VAs for RFCA, including idiopathic VT and PVC, between July 2010 and August 2018, 26 (5.9\%) patients were found to have an acute successful RFCA site at the AP-MA during the index procedure. None of these patients exhibited significant coronary artery disease by coronary angiography or CT coronary angiography and any structural heart disease. They failed beta-blocker or at least one anti-arrhythmic drug therapy. Monomorphic non-sustained VT (defined as three or more consecutive PVCs, was present in 3 patients, and monomorphic PVCs were seen in the remaining 23 patients. All patients were in normal sinus rhythm (SR) before RFCA. Twelve-lead ECG and 24-h ambulatory Holter were carried out at least once before RFCA. The demographic and clinical data, including patient age, sex, height, weight, biochemical blood examination results, echocardiographic parameters and clinical arrhythmias, were collected prior to the index procedure.

\section{ECG analysis}

Twelve-lead ECGs were recorded utilizing the Libang Electrical system (Libang ECG recording, Libang Medical, Shenzhen, China). The ECGs were analyzed at a paper speed of $25 \mathrm{~mm} / \mathrm{s}$, and the signals were amplified at $10 \mathrm{~mm} / \mathrm{mV}$. VAs were analyzed for the following parameters: (1) The QRS amplitude in the inferior leads; (2) The QRS width; (3) The maximum deflection index (MDI), defined as the duration from the earliest activation to the peak of the largest amplitude deflection divided by the total QRS 
duration, measured in the precordial leads; (4) The peak deflection index (PDI), defined as the duration from the earliest activation to the peak of the largest amplitude deflection divided by the total QRS duration, measured in the inferior leads; (5) The $S$-wave in lead $V_{6}$; and (6) Inferior lead notching. All parameters were measured with electronic calipers by 3 experienced investigators blinded to the site of origin. We adopted the mean values of these measurements as the data. If the interobserver difference was more than $5 \mathrm{~ms}$, the final decision was made by a joint meeting of the observers.

\section{Preparation before RFCA}

All antiarrhythmic drugs were discontinued at least 5 half-lives before the electrophysiological (EP) study. Intracardiac tracings were recorded utilizing a Prucka CardioLab ${ }^{\mathrm{TM}}$ electrophysiology system (General Electric Health Care System, Inc, Milwaukee, WI, USA). If the clinical VAs did not occur spontaneously and were not induced at baseline, intravenous isoproterenol (0.5 to 2.0 $\mathrm{g} / \mathrm{min}$ ) was administered to induce the clinical VAs. A 7.5-French, 3.5-mm-tip, irrigated ablation catheter (NaviStar ThermoCool, Biosense Webster, Diamond Bar, CA, USA) was then introduced into the left ventricle (LV) using a transaortic or transseptal approach, and intravenous heparin was administered to maintain an activated clotting time of 250 - 300 seconds.

\section{Electrogram (EGM) collection and analysis}

During an episode of clinical VA, activation mapping was performed. A minimum of three arrhythmic beats were recorded at the mapping site. The unipolar EGM was recorded from the distal (D) electrode of the mapping catheter and filtered at 0.5-100 $\mathrm{Hz}$. The bipolar EGM was recorded from the distal (D-2) electrode pairs of the mapping catheter and filtered at 30-500 Hz. All EGM and twelve-lead ECG data were stored on the multichannel mapping system for offline analysis with a paper speed of $100 \mathrm{~mm} / \mathrm{s}$ [5]. As shown in Figure 1, the morphological features can be used to determine the R-ratio, which is derived from the unipolar EGM as the amplitude of the first positive peak relative to that of the nadir (R-ratio). If no positive peak is present, then the R-ratio is 0. The QRS - Uni interval was calculated from the QRS onset to the maximum descending slope (D-Max) in the unipolar EGM. The V-QRS interval was calculated from the start of the bipolar ventricular EGM to the QRS onset. All parameters were measured with electronic calipers by 3 experienced investigators blinded to the site of origin. We adopted the mean values of these measurements as the data. If the interobserver difference was more than $5 \mathrm{~ms}$, the final decision was made by a joint meeting of the observers.

\section{Pace mapping}

Pace mapping was also performed at the earliest activation site using the distal bipolar electrodes at a coupling interval of the VA interval and a stimulus amplitude $1 \mathrm{~mA}$ greater than the late diastolic threshold (up to a maximum output of $10 \mathrm{~mA}$ and pulse width of $2.0 \mathrm{~ms}$ ). If present, a perfect pace-mapping match (12/12 leads) was defined as indicating the site of origin; otherwise, the activation mapping result was only used for guiding RFCA.

\section{RFCA}

RFCA were applied at the site where the earliest V-QRS, shortest QRS - Uni interval, or perfect pace-mapping match on ECG were recorded. RFCA was delivered using the power-control mode at a maximum power of 35 to $40 \mathrm{~W}$ and temperature of $43^{\circ} \mathrm{C}$ with irrigation mode at a flow rate of $17 \mathrm{~mL} / \mathrm{min}$. If the VAs were not eliminated within 30 seconds after energy delivery, the energy application was terminated, and the RFCA site was tagged as an unsuccessful site on the map. If the VAs were abolished within 30 seconds, the energy application was continued for a total of $300-500$ seconds, and the site was tagged as a successful site on the map.

\section{Definition of acute successful RFCA}

Acute successful RFCA was defined according to the following criteria: absence of spontaneous or induced clinical VAs, both in the absence and presence of isoproterenol infusion after RFCA with observation lasting 0.5 to 1 hour.

\section{Definition of AP-MA origin}


We defined an AP-MA location as follows: 1) the catheter tip demonstrated that the characteristic AP-MA location and motion when viewed on the left and right anterior oblique fluoroscopic views at the successful RFCA site; 2) the catheter tip could be curved and decurved freely on the left anterior oblique fluoroscopic view; 3) the ratio of atrial /ventricular EGMs at the RFCA site was $<1$, and the amplitudes of the atrial / ventricular EGMs were $>0.08 / 0.5 \mathrm{mV}$ at the RFCA site during sinus beat, respectively

[3]; 4) the catheter tip was on the left side of the AMC based on the CARTO3 map; 5) acute successful VAs elimination were achieved by RFCA energy delivery at the site; 6) the ECG showed a monophasic R-wave and no S-wave in the inferior leads during VA.

\section{Observation after RFCA and at the one-year follow up}

The patients were monitored for at least 3 days in the hospital after RFCA, and twelve-lead ECG and 24-hour ambulatory Holter monitoring were carried out at least once. The patients were followed in our outpatient arrhythmia clinic for one year after RFCA, and twelve-lead ECG and 24-hour ambulatory Holter monitoring were carried out at least once every three months. In addition, all patients were contacted by telephone at the time of manuscript preparation. Clinical success was defined as free of clinical VAs (symptomatic or asymptomatic) that were targeted during RFCA at the follow-up visit, and at least $80 \%$ reduction of VAs burden documented on post-RFCA 24-hour Holter recording as compared to pre-RFCA VAs burden.

\section{Statistical analysis}

Continuous data are given as the mean \pm SD. Noncontinuous data are given as the interquartile range and median in parentheses. A p value $<0.05$ was considered to indicate statistical significance.

\section{Results}

\section{Location and frequency of VAs}

Of 437 patients referred for RFCA of idiopathic VAs, twenty-six (5.9\%) patients were found to have an acute successful RFCA site at the AP-MA. The other origins were registered as follows: RVOT, 41.2\%; ASC, 30.2\%; AMC, 7.3\%; tricuspid annulus (TA), 6.9\%; papillary muscle, $3.2 \%$; lateral and posterior portion of the MA, $6.6 \%$; great cardiac vein and anterior inter-ventricular vein, $1.6 \%$; and other sites, $3 \%$. The mean age of the 26 patients with AP-MA VAs was $61 \pm 9$ years with 14 males and 12 females, and their clinical characteristics were summarized in Table 1. 
Table 1 Characteristics of the study population

\begin{tabular}{lr} 
& $\mathrm{N}=26$ \\
\hline Age (years) & $61 \pm 9(31-78)$ \\
Male sex & $14 / 26(53.8 \%)$ \\
BMI & $25.2 \pm 3.5$ \\
K & $4.03 \pm 0.37$ \\
Cr & $67.7 \pm 13.0$ \\
UA & $319.2 \pm 86.2$ \\
Glu & $4.7-5.8(5.2)^{*}$ \\
LDL-C & $3.08 \pm 0.80$ \\
TC & $3.9-5.4(5.0)^{*}$ \\
TG & $1.60 \pm 0.73$ \\
LA-D (mm) & $34.0-40.0(36.5)^{*}$ \\
LVEDD (mm) & $46.8-54.0(48.5)^{*}$ \\
LVEF (\%) before ablation & $56.0-65.0(60.5)^{*}$ \\
Clinical VAs & 23 \\
Only PVC & 3 \\
PVC, nonsustained VT &
\end{tabular}

Values are given as the mean \pm SD (range) or $\mathrm{n}(\%)$, unless otherwise indicated. BMI = body mass index. * Indicates the interquartile range and median in parentheses for non-normally distributed data, the same as in Table 2 and Table 3.

\section{ECG characteristics of VAs}

All patients exhibited a right bundle branch block, inferior-axis QRS morphology and negative QRS complexes in leads aVL and aVR. As shown in Table 2, the $R_{\square}, S_{\square}, R_{\square}, R_{\square}, Q_{a V R}, Q_{a V L}$, and $R_{a V F}$ amplitudes were $0.00-0.30(0.08) \mathrm{ms}, 0.50 \pm 0.17 \mathrm{~ms}, 1.50 \pm$ $0.47 \mathrm{~ms}, 1.66 \pm 0.54 \mathrm{~ms}, 0.60-0.89(0.80) \mathrm{ms}, 0.94 \pm 0.27 \mathrm{~ms}$, and $1.51 \pm 0.43 \mathrm{~ms}$, respectively. Inferior lead notching was recorded in 14 patients $(53.8 \%)$, and $S$-waves were present in lead $\mathrm{V}_{6}$ for 6 patients $(23.1 \%)$. The duration of the QRS complexes during VA was $168 \pm 18 \mathrm{~ms}$. The MDI was $0.54 \pm 0.07$, and the PDI was $0.52 \pm 0.11$. 


\begin{tabular}{|c|c|c|c|c|c|c|c|c|c|c|c|c|}
\hline \multirow[t]{2}{*}{ Pt. No } & \multicolumn{7}{|c|}{ QRS voltage in the inferior leads $(\mathrm{mV})$} & \multirow{2}{*}{$\begin{array}{c}\text { QRS } \\
\text { duration } \\
(\mathrm{ms})\end{array}$} & \multirow[t]{2}{*}{ MDI } & \multirow[t]{2}{*}{ PDI } & \multirow{2}{*}{$\begin{array}{c}\text { S- } \\
\text { waves } \\
\text { in } \\
\text { lead } \\
\mathrm{V}_{6}\end{array}$} & \multirow{2}{*}{$\begin{array}{c}\text { Inferior } \\
\text { lead } \\
\text { notching }\end{array}$} \\
\hline & $\mathrm{R}_{\mathrm{\square}}(\mathrm{mV})$ & $\mathrm{S}_{\square}(\mathrm{mV})$ & $\mathrm{R}_{\square}(\mathrm{mV})$ & $\mathrm{R}_{\square}(\mathrm{mV})$ & $\mathrm{Q}_{\mathrm{aVR}}(\mathrm{mV})$ & $\mathrm{Q}_{\mathrm{aVL}}(\mathrm{mV})$ & $\mathrm{R}_{\mathrm{aVF}}(\mathrm{mV})$ & & & & & \\
\hline 1 & 0.35 & 0.55 & 1.58 & 1.38 & 0.88 & 0.57 & 1.40 & 173 & 0.68 & 0.63 & no & yes \\
\hline 2 & 0.00 & 0.32 & 1.15 & 1.20 & 0.60 & 0.80 & 1.20 & 157 & 0.47 & 0.46 & no & no \\
\hline 3 & 0.28 & 0.82 & 1.25 & 1.10 & 0.77 & 0.70 & 1.17 & 157 & 0.54 & 0.57 & no & yes \\
\hline 4 & 0.00 & 0.68 & 1.95 & 2.14 & 1.12 & 1.32 & 2.14 & 158 & 0.51 & 0.52 & no & yes \\
\hline 5 & 0.10 & 0.40 & 1.00 & 1.25 & 0.50 & 0.70 & 1.10 & 147 & 0.76 & 0.74 & yes & yes \\
\hline 6 & 0.00 & 0.30 & 0.80 & 0.90 & 0.30 & 0.60 & 0.80 & 192 & 0.55 & 0.33 & no & yes \\
\hline 7 & 0.00 & 0.71 & 2.52 & 3.10 & 1.35 & 1.48 & 2.17 & 198 & 0.31 & 0.61 & no & yes \\
\hline 8 & 0.00 & 0.70 & 1.02 & 1.18 & 0.75 & 0.75 & 1.07 & 172 & 0.63 & 0.66 & yes & yes \\
\hline 9 & 0.25 & 0.35 & 1.60 & 1.80 & 0.80 & 1.00 & 1.60 & 183 & 0.52 & 0.52 & no & no \\
\hline 10 & 0.40 & 0.62 & 2.34 & 2.27 & 1.39 & 0.9 & 1.42 & 142 & 0.54 & 0.54 & no & yes \\
\hline 11 & 0.35 & 0.40 & 1.30 & 1.20 & 0.80 & 0.8 & 0.90 & 147 & 0.58 & 0.67 & no & no \\
\hline 12 & 0.05 & 0.07 & 1.30 & 1.30 & 0.60 & 0.9 & 1.30 & 183 & 0.56 & 0.33 & yes & yes \\
\hline 13 & 0.00 & 0.50 & 1.50 & 1.80 & 0.80 & 1.00 & 1.60 & 186 & 0.58 & 0.40 & yes & yes \\
\hline 14 & 0.00 & 0.48 & 1.45 & 1.85 & 0.75 & 0.90 & 1.55 & 181 & 0.51 & 0.40 & no & yes \\
\hline 15 & 0.42 & 0.51 & 1.91 & 2.24 & 1.09 & 1.27 & 2.06 & 170 & 0.55 & 0.64 & no & no \\
\hline 16 & 0.00 & 0.45 & 1.80 & 2.10 & 0.80 & 1.20 & 2.00 & 158 & 0.43 & 0.49 & no & yes \\
\hline 17 & 0.30 & 0.60 & 1.00 & 1.60 & 0.80 & 1.00 & 1.60 & 150 & 0.59 & 0.65 & no & no \\
\hline 18 & 0.10 & 0.50 & 1.90 & 2.15 & 0.80 & 1.30 & 2.05 & 145 & 0.59 & 0.62 & no & no \\
\hline 19 & 0.40 & 0.71 & 2.30 & 2.20 & 1.05 & 1.40 & 2.40 & 165 & 0.58 & 0.53 & no & no \\
\hline 20 & 0.28 & 0.48 & 1.30 & 1.60 & 0.80 & 0.9 & 1.50 & 167 & 0.45 & 0.46 & yes & yes \\
\hline 21 & 0.03 & 0.35 & 1.23 & 1.00 & 0.73 & 0.47 & 1.13 & 156 & 0.58 & 0.52 & no & no \\
\hline 22 & 0.00 & 0.63 & 1.41 & 1.51 & 0.78 & 0.88 & 1.56 & 186 & 0.59 & 0.40 & no & no \\
\hline 23 & 0.05 & 0.54 & 1.13 & 0.93 & 0.60 & 0.63 & 1.02 & 136 & 0.59 & 0.53 & no & no \\
\hline 24 & 0.30 & 0.60 & 0.90 & 1.45 & 0.40 & 1.00 & 1.15 & 178 & 0.53 & 0.49 & no & yes \\
\hline 25 & 0.15 & 0.22 & 2.12 & 2.33 & 0.9 & 0.85 & 1.85 & 191 & 0.42 & 0.42 & no & no \\
\hline 26 & 0 & 0.41 & 1.32 & 1.65 & 0.45 & 1.05 & 1.40 & 195 & 0.50 & 0.46 & yes & no \\
\hline Mean \pm & $0.00-$ & $0.50 \pm$ & $1.50 \pm$ & $1.66 \pm$ & $0.60-$ & $0.94 \pm$ & $1.51 \pm$ & $168 \pm$ & 0.54 & 0.52 & $23.1 \%$ & $53.8 \%$ \\
\hline $\begin{array}{l}\text { SD or } \\
\text { percent }\end{array}$ & $\begin{array}{c}0.30 \\
(0.08)^{*}\end{array}$ & 0.17 & 0.47 & 0.54 & $\begin{array}{c}0.89 \\
(0.80)^{*}\end{array}$ & 0.27 & 0.43 & 18 & $\begin{array}{c} \pm \\
0.07\end{array}$ & $\begin{array}{c} \pm \\
0.11\end{array}$ & & \\
\hline
\end{tabular}

Values are given as the mean \pm SD (range) or percent (\%), unless otherwise indicated. The maximum deflection index (MDI) was defined as the duration from the earliest activation to the peak of the largest amplitude deflection divided by the total QRS duration, measured in the precordial leads. (4) The peak deflection index (PDI) was defined as the duration from the earliest activation to the peak of the largest amplitude deflection divided by the total QRS duration, measured in the inferior leads. 
Coronary angiography and coronary venous angiography were performed prior to mapping. Detailed mapping of the ASC, AMC, distal portion of the coronary venous, AP-MA regions were performed in all the 26 patients. No areas of abnormal endocardial voltage were seen in the AP-MA regions. In 437 patients treated by RFCA, acute successful RFCA at the AP-MA were achieved in 26 (5.9\%) (25 patients with a transaortic approach, 1 patient with a transseptal approach). As shown in Table 3, the A/V amplitude ratio at the RFCA site during SR was $0.23 \pm 0.15$. The QRS - Uni interval in the unipolar EGM was $18.8 \pm 13.6 \mathrm{~ms}$. The R-ratio in the unipolar EGM was $0.00-0.03(0.00)$ at the successful RFCA site, and initial unipolar QS-waves were recorded in 19 patients (73.1\%). With bipolar mapping, the V-QRS interval was $3.75-17.3(11) \mathrm{ms}, 6(23.1 \%)$ patients showed earliest V-QRS interval of $0 \mathrm{~ms}$, and the other 20 (76.9\%) patients showed V-QRS interval of 10-54 ms. A presystolic potential that preceding the QRS onset was recorded during VA in 1 patient (3.8\%). Figure $\mathbf{2 G}$ and $\mathbf{3 D}$ showed earliest V-QRS interval of $0 \mathrm{~ms}$ and $31 \mathrm{~ms}$ for bipolar recording during VAs, respectively. 
Table 3 Electrophysiologic Characteristics and ablation result of AP-MA VAs

\begin{tabular}{|c|c|c|c|c|c|c|c|c|c|}
\hline Pt. No & $\begin{array}{l}\text { Pre- } \\
\text { potential }\end{array}$ & $\begin{array}{l}\text { A/V ratio } \\
\text { during } \\
\text { sinus } \\
\text { rhythm }\end{array}$ & $\begin{array}{l}\mathrm{R} / \mathrm{S} \text { ratio } \\
\text { during } \\
\text { unipolar } \\
\text { recording }\end{array}$ & $\begin{array}{l}\text { QRS- } \\
\text { Uni } \\
\text { (ms) }\end{array}$ & $\begin{array}{c}\text { Perfect } \\
\text { pace } \\
\text { match }\end{array}$ & $\begin{array}{l}\text { V-QRS } \\
\text { interval } \\
\text { (ms) }\end{array}$ & $\begin{array}{l}\text { RFCA } \\
\text { start } \\
\text { to } \\
\text { effect } \\
\text { (s) }\end{array}$ & $\begin{array}{c}\text { Recurrence } \\
\text { during } 3 \\
\text { days of } \\
\text { monitoring }\end{array}$ & $\begin{array}{c}\text { Recurrence } \\
\text { during } 1 \\
\text { year of } \\
\text { follow up }\end{array}$ \\
\hline 1 & no & 0.21 & 0.19 & 52 & no & 54 & 8.1 & yes & no \\
\hline 2 & no & 0.28 & 0.00 & 0 & no & 0 & 17.3 & no & yes \\
\hline 3 & no & 0.35 & 0.31 & 7 & no & 15 & 7.7 & no & no \\
\hline 4 & no & 0.03 & 0.22 & 47 & no & 18 & 4.2 & no & no \\
\hline 5 & no & 0.05 & 0.00 & 6 & no & 31 & 16.5 & no & no \\
\hline 6 & no & 0.58 & 0.09 & 30 & no & 0 & 17.3 & no & no \\
\hline 7 & no & 0.03 & 0.00 & 25 & no & 5 & 30.2 & no & yes \\
\hline 8 & no & 0.16 & 0.00 & 12 & yes & 7 & $\mathrm{~N} / \mathrm{A}$ & no & no \\
\hline 9 & no & 0.46 & 0.00 & 24 & yes & 50 & 19.3 & no & yes \\
\hline 10 & no & 0.04 & 0.00 & 8 & yes & 12 & 9.2 & no & no \\
\hline 11 & no & 0.31 & 0.00 & 22 & no & 0 & 5.59 & no & no \\
\hline 12 & no & 0.10 & 0.10 & 16 & no & 14 & 21.3 & no & no \\
\hline 13 & yes & 0.43 & 0.07 & 40 & no & 0 & 17.4 & yes & no \\
\hline 14 & no & 0.33 & 0.00 & 26 & no & 17 & 19.1 & yes & yes \\
\hline 15 & no & 0.05 & 0.00 & 17 & no & 14 & 17.5 & no & no \\
\hline 16 & no & 0.21 & 0.00 & 12 & yes & 0 & 3.2 & yes & no \\
\hline 17 & no & 0.43 & 0.00 & 8 & no & 7 & 20.1 & no & no \\
\hline 18 & no & 0.04 & 0.00 & 15 & no & 0 & 23.1 & yes & yes \\
\hline 19 & no & 0.22 & 0.00 & 28 & yes & 9 & 21.7 & no & no \\
\hline 20 & no & 0.22 & 0.00 & 24 & yes & 12 & 14.4 & no & no \\
\hline 21 & no & 0.19 & 0.00 & 9 & yes & 10 & 5.6 & no & no \\
\hline 22 & no & 0.13 & 0.00 & 0 & yes & 9 & 13.2 & no & no \\
\hline 23 & no & 0.28 & 0.00 & 0 & yes & 14 & 5.4 & yes & yes \\
\hline 24 & no & 0.29 & 0.00 & 24 & no & 6 & $\mathrm{~N} / \mathrm{A}$ & no & yes \\
\hline 25 & no & 0.28 & 0.00 & 12 & no & 37 & 8.5 & no & no \\
\hline 26 & no & 0.23 & 0.02 & 25 & no & 25 & $\mathrm{~N} / \mathrm{A}$ & no & no \\
\hline mean \pm & $3.8 \%$ & $0.23 \pm$ & $0.00-0.03$ & $18.8 \pm$ & $34.6 \%$ & $3.75-$ & $14.1 \pm$ & $23.1 \%$ & $26.9 \%$ \\
\hline $\begin{array}{l}\text { SD or } \\
\text { percent }\end{array}$ & & 0.15 & $(0.00) *$ & 13.6 & & $\begin{array}{c}17.3 \\
(11)^{*}\end{array}$ & 7.2\# & & \\
\hline
\end{tabular}


Values are given as the mean \pm SD (range) or percent (\%), unless otherwise indicated. N/A: Time to effect were not available due to infrequently episode of VAs during ablation. \#: The RFCA start-to-effect time of $14.1 \pm$ 7.2 seconds were for the 23 patients (88.5\%), in the remaining 3 patients (11.5\%), the mean duration of successful RFCA was not well determined due to infrequent nature of clinical VAs during ablation.

\section{Pace mapping}

Characteristics of the QRS morphology during pacing in 9 patients (34.6\%) were almost identical to those of the VAs successfully ablated at the AP-MA. An interval of 26 ms between the pacing stimulus artifact and QRS onset was recorded in 1 patient (Figure 2C).

\section{RFCA at the AP-MA}

Complete elimination of the VA could be achieved by RFCA at the RFCA site where the earliest V-QRS interval or shortest QRS Uni interval were recorded at the AP-MA. The AP-MA VAs RFCA location were shown in Figure 2E/2F and 3F/3G (Right and left anterior oblique fluoroscopic views). The RFCA start-to-effect time was $14.1 \pm 7.2$ seconds in 23 patients (88.5\%). In the remaining 3 patients $(11.5 \%)$, the mean duration of successful RFCA was not well determined due to infrequent nature of clinical VAs during RFCA. No complications occurred during the RFCA procedure.

\section{Observations after RFCA and at the one-year follow up}

Clinical VAs could still be recorded in 6 patients (23.1\%) during 3 days of in-hospital monitoring after RFCA, the VA disappeared in $7.7 \%(2 / 26)$ of patients 3 days later due to delayed RFCA efficacy, and during the one-year follow-up period, $26.9 \%(7 / 26)$ of patients had clinical VA recurrence, and one patient undergo re-do procedure. The final RFCA target was the same as the index procedure based on X-ray fluoroscopic views and CARTO3 mapping result.

\section{Discussion}

The current study has four major findings. First, the frequency of AP-MA VAs confirmed by successful RFCA was $5.9 \%$ in 437 consecutive patients with idiopathic VAs in a single center. Second, the QRS - Uni interval could work as a marker for guiding RFCA and help reduce unnecessary RFCA applications. Third, 6 (23.1\%) patients showed the earliest bipolar activation preceded the QRS onset of $0 \mathrm{~ms}$ at the acute successful RFCA site. Forth, RFCA is effective for the acute elimination of these AP-MA VAs.

The EP features of the AP-MA VAs include preferential conduction, multiple exits, ventricular prepotentials, and a long stimulusto-QRS interval with pacing (Figure 2C). These EP features and the anatomical complexity of the AP-MA VAs may present challenges during the mapping and RFCA of VAs arising from this region [6]. Our results indicate that VAs with acute successful RFCA at the AP-MA can be identified as one subgroup of VAs with distinctive ECG characteristics and RFCA is effective for the acute elimination of these VAs.

The location of the RFCA target were located in posterior portion of the left ventricle, and the myocardium at the RFCA target depolarized in the direction that toward these precordial electrodes. This could explain the reason that the precordial transition of $R / S>1$ in lead $V_{1}$ in most of these cases during VAs. The timing of excitation of left and right ventricles is considered to mainly affect the QRS morphology and the duration in the inferior leads. When VAs originate near the AMC of the AP-MA, the ventricular septum and right ventricle would be depolarized earlier than VAs originating from the free wall of the AP-MA, this may result in the absence of notching in the inferior leads or the absence of an S-wave in lead $V_{6}$.

As shown in Table 2, QRS complexes during VAs all showed right bundle branch block pattern, S-waves were present in lead $\mathrm{V}_{6}$ in 6 patients $(23.1 \%)$, and inferior lead notching was present in 14 patients (53.8\%). Delta-wave-like onset of the precordial QRS complex during VAs may support the theory that the VAs originated from deep inside the sub-endocardium or epicardium at the AP-MA (Figure 2A) $[8,9]$. It is likely that some VAs might have overlapped with the VAs that originated from the AMC out of all the 
other origins [10,11]. Li et al reported the r-wave amplitude in lead $V_{1}$ was greater than that in lead $V_{2}$ in 4 of 5 patients with VAs arising from proximal portion of the anterior inter-ventricular vein [12]. Kumagai et al reported AP-MA VAs had an Rs pattern in some precordial leads except for lead $V_{6}$ [13]. A notched QRS pattern and a longer QRS duration in inferior leads may indicate VAs originating from the free wall of the AP-MA in our study (Figure 2A).

Although several studies have reported the successful ablation of VAs near the AP-MA, none have systemically reported the prevalence, ECG and EP characteristics of VAs originating from the AP-MA and analyzed the follow-up results in as many patients as in this study [2,3]. The AP-MA is close to the posterior portion of the RVOT, the LV summit myocardium near the left sinus cusp, and the sub-valvular portion of the LVOT, which are reported as foci of VAs originating from the LVOT/RVOT [6,7]. The close anatomic proximity suggests that different forms of VAs could likely originate from one single origin, or activation of alternative pathways between the VA focus and an exit point [4].

Kumagai et al reported VAs arose from the mitral annulus had a delta wave-like QRS morphology and was sensitive to isoproterenol and ATP, but insensitive to programmed pacing or burst pacing [14]. These findings support a mechanism of triggered activity rather than a reentrant mechanism. Anderson et al identified histologically and histochemically different AV ring-specialized tissue at the AP-MA [15]. We hypothesize that the target site around the AP-MA may reflect the activation of a segment of $A V$ ring-specialized tissue that is poorly coupled with the surrounding ventricular myocardium.

Conventionally, the strategy to guide RFCA in the treatment of VAs is based on activation mapping during bipolar recording and the presence of QS-waves during unipolar recording [16-18]. In this study, complete initial unipolar QS-waves were recorded in 18 patients (69.2\%). The QRS - Uni interval may be related to how fast the wave front conducts from the recording location of the mapping catheter to the breakout point, this could work as a marker for guiding RFCA. As shown in Table 3, with bipolar mapping, the V-QRS interval was 3.75 - $17.3(11) \mathrm{ms}, 6(23.1 \%)$ patients showed earliest V-QRS interval of 0 ms, and the other 20 patients $(76.9 \%)$ showed V-QRS interval of 10-54 ms. Excellent leading V-potentials from the successful RFCA site could not be identified in more than half of these cases, indicating intramural or epicardial origin of these VAs.

Successful catheter RFCA was achieved underneath the AP-MA using a transaortic approach in 25 cases (96.2\%); in one case, RFCA could be achieved only by using a transseptal approach due to the anatomical complexity of the AP-MA. The RFCA startto-effect time was $14.1 \pm 7.2$ seconds in 23 patients (88.5\%). In the remaining 3 patients $(11.5 \%)$, the mean duration of successful RFCA was not well determined due to infrequent nature of clinical VAs during RFCA. Clinical VAs could still be recorded in 6 patients $(23.1 \%)$ during 3 days of in-hospital monitoring after RFCA, and 7.7\% (2/26) of VAs disappeared 3 days later due to delayed RFCA efficacy, which indicates that waiting for delayed RFCA efficacy is also a reasonable choice in cases of VAs arising from the region of the AP-MA [19].

Epicardial RFCA via the transpericardial approach is not available for the treatment of idiopathic VAs originating from the left ventricular (LV) summit because of the anatomic barriers such as the close proximity to the coronary arteries, thick epicardial fat pads, and (or) not reachable due to anatomical limitations [20]. The sites of VAs origins from the LV summit area are roughly predicted by the information from mapping in the corresponding endocardial AP-MA, ASC, AMC or other areas on the assumption that the conduction properties in these regions are normal. Komatsu et al reported mapping the LV summit by using a micro-catheter is feasible and helpful for identifying the site of origin. In his study, it was not possible to advance a standard irrigated-tip ablation catheter to the LV summit area, and ablation was attempted at adjacent structures [21]. Therefore, RFCA at near sites, such as the AP-MA is one of the alternative ablation technique to treat these VAs in our study. Recognition of these possibilities is helpful for RFCA of such VAs originating from the AP-MA.

There are limitations to this retrospective study. First, the exact origins of VAs may originate from adjacent origins, including the anterior inter-ventricular vein, great cardiac vein and other epicardial locations but can't be confirmed or ablated because the ideal target sites were not reachable due to anatomical limitations. Second, intra-cardiac echocardiography was not used to confirm the RFCA target. Therefore, the exact origins of VAs could not be ascertained. Third, the target site might not precisely coincide with, although we believe that is very near, the VA origin. Forth, while the potential preceding the QRS complexes was recorded at this site of successful RFCA, its significance and genesis were not determined. Further evaluation with a prospective study design is needed to assess the reproducibility of the AP-MA VAs.

Page $10 / 15$ 


\section{Conclusions}

VAs with acute successful RFCA at the AP-MA are a rare but identifiable sub-group of VAs with distinctive EP and ECG characteristics. RFCA is effective for the acute elimination of these VAs. Advanced knowledge of the AP-MA anatomy, ECG and EP features may be useful in planning and/or facilitating the RFCA procedure. Aside from the bipolar V-QRS interval, the QRS Uni interval could work as a marker for guiding RFCA and help reduce unnecessary RFCA applications.

\section{Abbreviations}

Vas: Ventricular arrhythmias; VT: Ventricular tachycardia; PVC: Premature ventricular contractions; RFCA: Radiofrequency catheter ablation; AP-MA: Anterior portion of the mitral annulus: EGM: Electrogram; RVOT: Right ventricular outflow tract; LVOT: Left ventricular outflow tract; ASC: Aortic sinus cusp; AMC: Aortomitral continuity; ECG: Electrocardiogram; EP: Electrophysiological; SR: Sinus rhythm; MDI: Maximum deflection index; PDI: Peak deflection index; LV: Left ventricle; TA: Tricuspid annulus;

\section{Declarations}

Ethics approval and consent to participatelThe study was granted approval by the Central Ethics Committee of TEDA International Cardiovascular Hospital. Written informed consent from all participants was obtained.

Consent to publishपAll coauthors have seen and agree with the contents of the manuscript. Neither the entire paper nor any part of its content has not been under simultaneous consideration elsewhere and has not been previously published in similar form.

\section{Availability of data and materials}

The datasets used and/or analysed during the current study are de-identified and available from the corresponding author on reasonable request.

Competing interests!The authors declare that they have no competing interests.

Funding: None

Authors' contributions $\square \mathrm{DC}$ takes responsibility for all aspects of the reliability and freedom from bias of the data presented and their discussed interpretation. KL take responsibility for reviewing the manuscript. GP, WQ and WY take responsibility for data collection. All authors read and approved the final manuscript.

Acknowledgements: None

\section{Author details $\square$}

1 Cardiac Electrophysiology Unit, First Department of Cardiology, TEDA International Cardiovascular Hospital, Tianjin, China;

2 College of Clinical Cardiology, Tianjin Medical University, Tianjin, China $\rrbracket$

3 Cardiovscular Institute, Tianjin University, Tianjin, China》

4 Second Department of Cardiology, Laboratory of Cardiac Electrophysiology, Evangelismos General Hospital of Athens, 10676, Athens, Greece.

\section{References}

1. Cronin EM, Bogun FM, Maury P, Peichl P, Chen M, Namboodiri N, Aguinaga L, Leite LR, Al-Khatib SM, Anter E, et al. 2019 HRS/EHRA/APHRS/LAHRS expert consensus statement on catheter ablation of ventricular arrhythmias: Executive summary. Heart Rhythm. 2020;17(1):e155-e205.

Page $11 / 15$ 
2. Tada H, Ito S, Naito S, Kurosaki K, Kubota S, Sugiyasu A, Tsuchiya T, Miyaji K, Yamada M, Kutsumi Y, et al. Idiopathic ventricular arrhythmia arising from the mitral annulus: a distinct subgroup of idiopathic ventricular arrhythmias. J Am Coll Cardiol. 2005;45(6):877-886.

3. Wasmer K, Köbe J, Dechering DG, Bittner A, Pott C, Mönnig G, Milberg P, Frommeyer G, Zellerhoff S, Kochhäuser S, et al. Ventricular arrhythmias from the mitral annulus: patient characteristics, electrophysiological findings, ablation, and prognosis. Heart Rhythm. 2013;10(6):783-788.

4. Mountantonakis SE, Vaishnav AS, Jacobson JD, Bernstein NE, Bhasin K, Coleman KM, Skipitaris NT. Conduction patterns of idiopathic arrhythmias from the endocardium and epicardium of outflow tracts: New insights with noninvasive electroanatomic mapping. Heart Rhythm. 2019; 16(10):1562-1569.

5. Niu G, Feng T, Jiang C, Suo N, Lin J, Qu F, McSpadden LC, Yao Y, Zhang S. Predictive value of unipolar and bipolar electrograms in idiopathic outflow tract ventricular arrhythmia mapping and ablation. J Cardiovasc Electrophysiol. 2018;29(6):900-907.

6. Cheung JW, Anderson RH, Markowitz SM, Lerman BB. Catheter Ablation of Arrhythmias Originating From the Left Ventricular Outflow Tract. JACC Clin Electrophysiol. 2019;5(1):1-12.

7. Di Biase L, Romero J, Zado ES, Diaz JC, Gianni C, Hranitzki PM, Sanchez JE, Mohanty S, Al-Ahmad A, Mohanty P, et al. Variant of ventricular outflow tract ventricular arrhythmias requiring ablation from multiple sites: Intramural origin. Heart Rhythm. 2019;16(5):724-732.

8. Yamada T, Doppalapudi H, Litovsky SH, McElderry HT, Kay GN. Challenging Radiofrequency Catheter Ablation of Idiopathic Ventricular Arrhythmias Originating From the Left Ventricular Summit Near the Left Main Coronary Artery. Circ Arrhythm Electrophysiol. 2016; 9(10):e004202.

9. Yamada T, Yoshida N, Doppalapudi H, Litovsky SH, McElderry HT, Kay GN. Efficacy of an Anatomical Approach in Radiofrequency Catheter Ablation of Idiopathic Ventricular Arrhythmias Originating From the Left Ventricular Outflow Tract. Circ Arrhythm Electrophysiol. 2017;10(5):e004959.

10. Chen J, Hoff PI, Rossvoll O, De Bortoli A, Solheim E, Sun L, Schuster P, Larsen T, Ohm OJ. Ventricular arrhythmias originating from the aortomitral continuity: an uncommon variant of left ventricular outflow tract tachycardia. Europace. 2012;14(3):388-395.

11. Mizobuchi M, Enjoji Y. Demonstration of a hidden interaction between the aortomitral continuity and the conduction system in a case of idiopathic left ventricular outflow tract ventricular tachycardia. J Arrhythm. 2015;31(3):180-182.

12. Li YC, Lin JF, Li J, Ji KT, Lin JX. Catheter ablation of idiopathic ventricular arrhythmias originating from left ventricular epicardium adjacent to the transitional area from the great cardiac vein to the anterior interventricular vein. Int $\mathrm{J}$ Cardiol. 2013;167(6):2673-2681.

13. Kumagai K, Fukuda K, Wakayama Y, Sugai Y, Hirose M, Yamaguchi N, Takase K, Yamauchi Y, Takahashi A, Aonuma K, et al. Electrocardiographic characteristics of the variants of idiopathic left ventricular outflow tract ventricular tachyarrhythmias. $J$ Cardiovasc Electrophysiol. 2008;19(5):495-501.

14. Kumagai K, Yamauchi Y, Takahashi A, Yokoyama Y, Sekiguchi Y, Watanabe J, lesaka Y, Shirato K, Aonuma K. Idiopathic left ventricular tachycardia originating from the mitral annulus. J Cardiovasc Electrophysiol. 2005;16(10):1029-1036.

15. Anderson RH. Clinical anatomy of the aortic root. Heart. 2000;84(6):670-673.

16. Sousa PA, Elvas L, Barra S, António N, Gonçalves L. Multielectrode Mapping and Pattern Matching Recognition for Left Premature Ventricular Contraction Ablation. JACC Clin Electrophysiol. 2019;5(4):528-529.

17. Nakashima T, Kubota T, Takasugi N, Kawasaki M. Premature ventricular contraction eliminated by radiofrequency catheter ablation from the pulmonary sinus cusp using the ventricular potentials in the summit communicating vein as a guide for mapping. J Cardiovasc Electrophysiol. 2018;29(8):1169-1170.

18. Yamada T, McElderry HT, Doppalapudi H, Murakami Y, Yoshida Y, Yoshida N, Okada T, Tsuboi N, Inden Y, Murohara T, et al. Idiopathic ventricular arrhythmias originating from the aortic root prevalence, electrocardiographic and electrophysiologic characteristics, and results of radiofrequency catheter ablation. J Am Coll Cardiol. 2008;52(2):139-147.

Page $12 / 15$ 
19. Ding L, Hou B, Wu L, Qiao Y, Sun W, Guo J, Zheng L, Chen G, Zhang L, Zhang S, et al. Delayed efficacy of radiofrequency catheter ablation on ventricular arrhythmias originating from the left ventricular anterobasal wall. Heart Rhythm 2017;14(3):341-349.

20. Yamada T, Kumar V, Yoshida N, Doppalapudi H. Eccentric Activation Patterns in the Left Ventricular Outflow Tract during Idiopathic Ventricular Arrhythmias Originating From the Left Ventricular Summit. Circ Arrhythm Electrophysiol. 2019;12(8):e007419.

21. Komatsu Y, Nogami A, Shinoda Y, Masuda K, Machino T, Kuroki K, Yamasaki H, Sekiguchi Y, Aonuma K. Idiopathic Ventricular Arrhythmias Originating From the Vicinity of the Communicating Vein of Cardiac Venous Systems at the Left Ventricular Summit. Circ Arrhythm Electrophysiol. 2018;11(1):e005386.

\section{Figures}

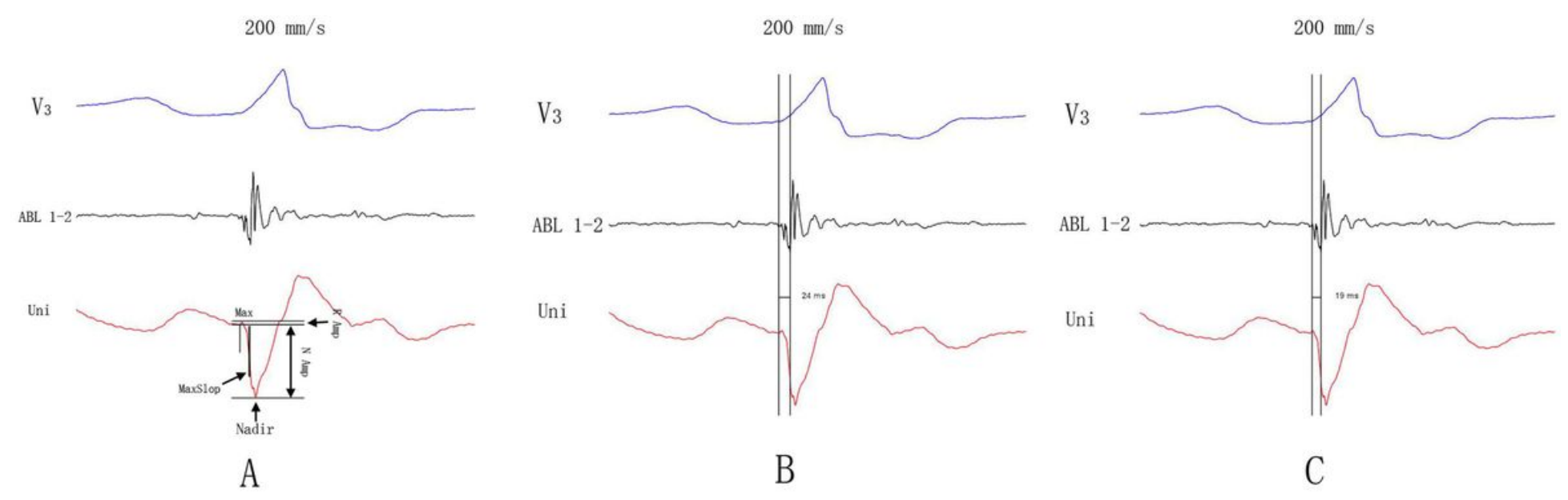

Figure 1

Illustration of key quantitative features on a sample bipolar and unipolar electrogram (RGM). R_amp, the amplitude of the first positive peak of the unipolar EGM; N_amp, the amplitude of the nadir of the unipolar EGM; R-ratio, the amplitude of the first positive peak relative to that of the nadir; MaxSlope, the maximum descending slope of the Q-wave; D-Max, the time interval between the initial descent point to the MaxSlope. The QRS - Uni interval was calculated from the QRS onset to the maximum descending slope (D-Max) of the unipolar EGM. The V-QRS interval was calculated from the start of the bipolar ventricular EGM to the QRS onset. 


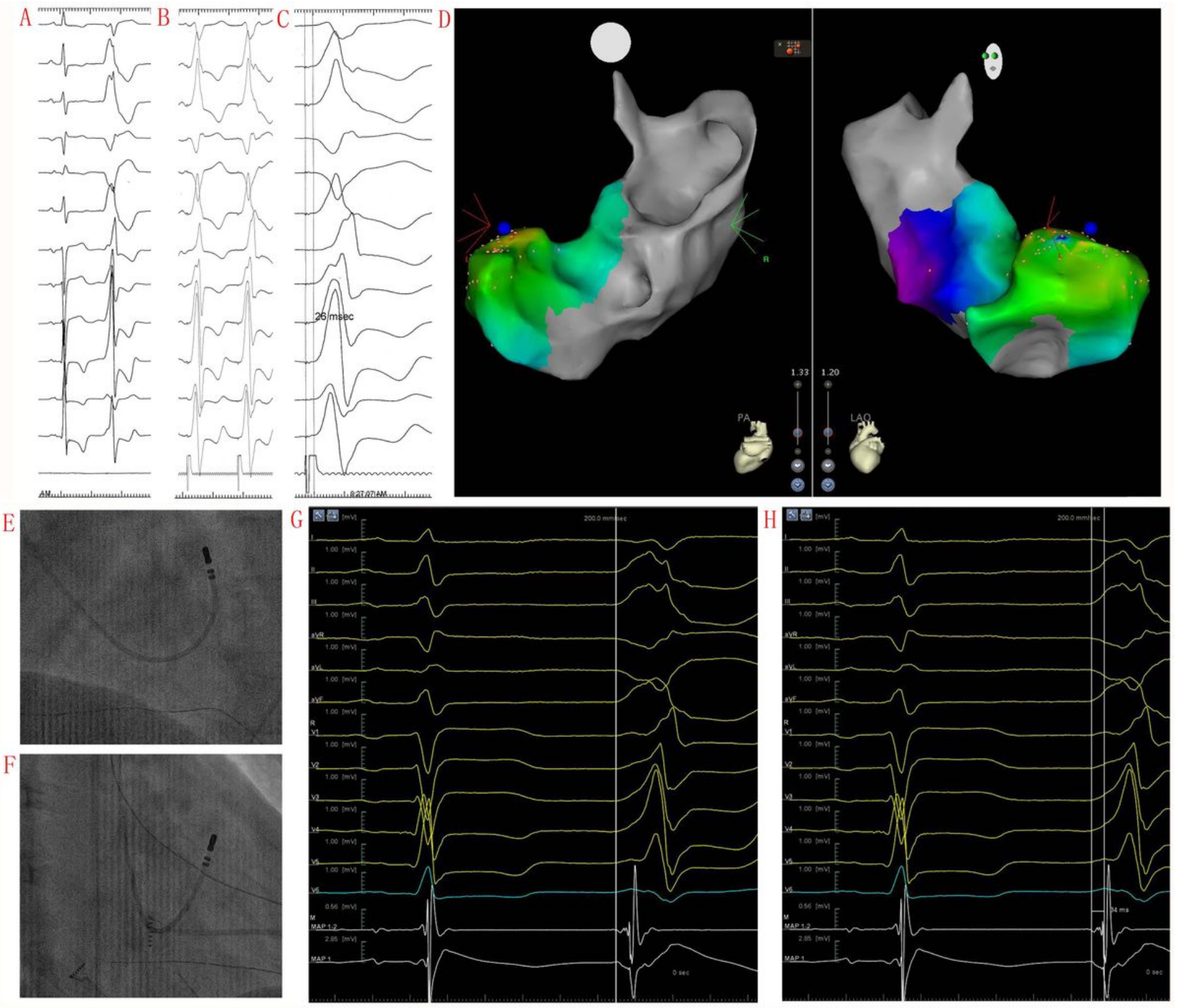

\section{Figure 2}

Patient No.13, premature ventricular contractions (PVCs) with acute successful RFCA at the anterior portion of the mitral annulus (AP-MA). A: Twelve-lead electrocardiographic (ECG) morphology of the QRS complex during sinus rhythm (SR) and PVCs (paper speed $25 \mathrm{~mm} / \mathrm{s}$ ). B: Pace-mapping QRS complex morphology (paper speed $25 \mathrm{~mm} / \mathrm{s}$ ). C: A stimulus-to-QRS interval of $26 \mathrm{~ms}$ with an excellent pace map was recorded at the acute successful RFCA site (paper speed $100 \mathrm{~mm} / \mathrm{s}$ ). D: CARTO3 mapping indicates an acute successful RFCA site at the AP-MA. E and F: Right and left anterior oblique fluoroscopic views indicate an acute successful RFCA site at the free wall of the AP-MA. G: Earliest V-QRS interval of 0 ms for bipolar recording during PVC (paper speed $100 \mathrm{~mm} / \mathrm{s}$ ) and an A/V ratio of 0.43 during SR. H: QRS - Uni interval of $34 \mathrm{~ms}$ for unipolar recording during PVC, with an R-ratio of 0.07 (paper speed $100 \mathrm{~mm} / \mathrm{s}$ ). 

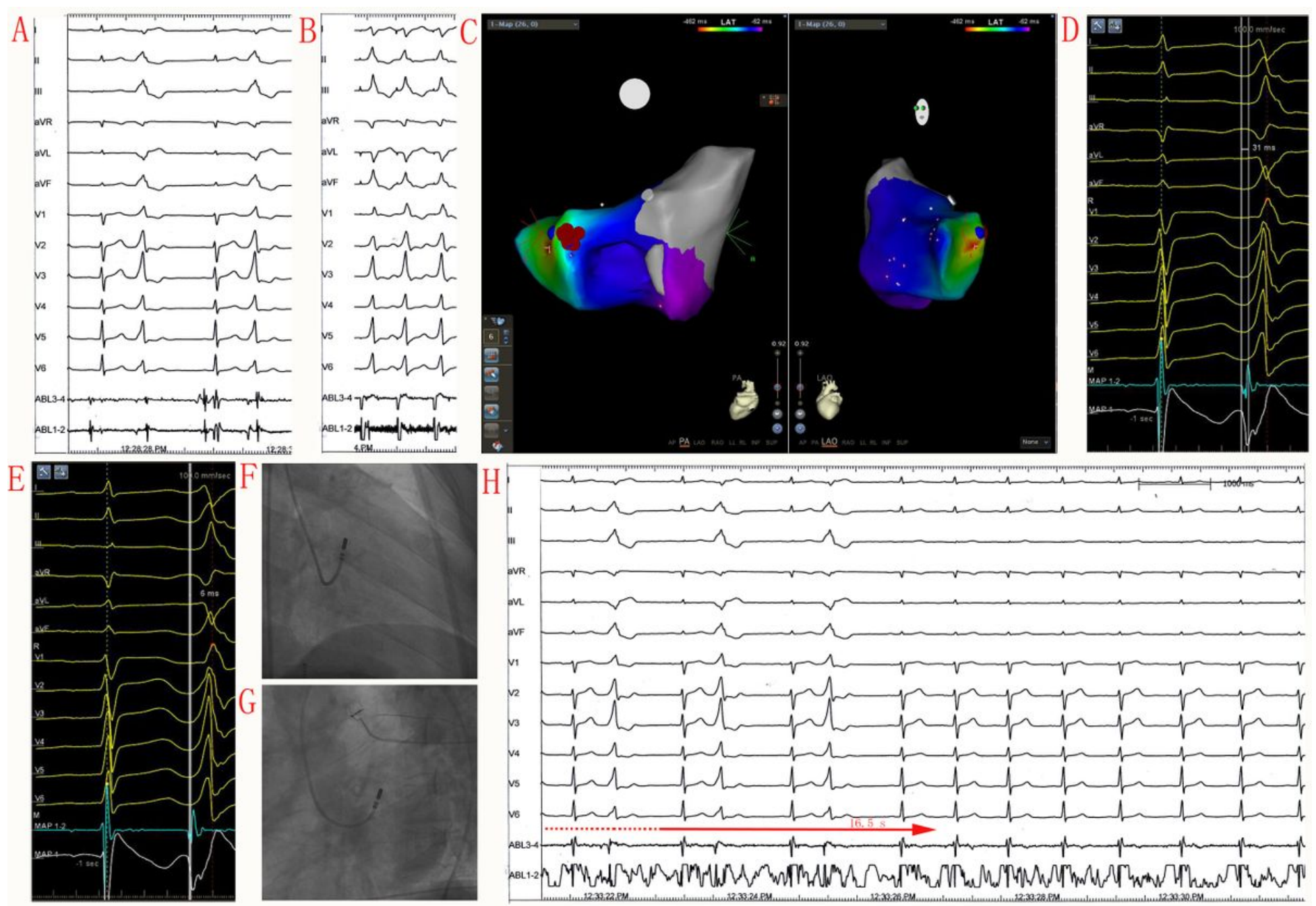

\section{Figure 3}

Patient No.5, PVCs with acute successful RFCA at the AP-MA. A: Twelve-lead ECG morphology of the QRS complex during SR and PVCs (paper speed $25 \mathrm{~mm} / \mathrm{s}$ ). B: Pace-mapping QRS complex morphology (paper speed $25 \mathrm{~mm} / \mathrm{s}$ ). C: CARTO3 mapping indicates an acute successful RFCA site at the AP-MA. D: Earliest V-QRS interval of $31 \mathrm{~ms}$ for bipolar recording during PVC and an A/V ratio during SR of 0.05 (paper speed $100 \mathrm{~mm} / \mathrm{s}$ ). G: QRS - Uni interval of $6 \mathrm{~ms}$ for unipolar recording during PVC and an R-ratio of 0 (paper speed $100 \mathrm{~mm} / \mathrm{s}$ ). F and G: Right and left anterior oblique fluoroscopic views at the AP-MA. H: Elimination of the PVCs after $16.5 \mathrm{~s}$ RFCA application. 